\title{
ANALYZING COMBINING ABILITY AND HETEROSIS OF THERMO- PHOTO SENSITIVE GENIC MALE STERILE WHEAT LINES FOR HYBRID DEVELOPMENT
}

\author{
Sedhom ABDELKHALIK ${ }^{1,2}$, Ding MINGLIANG ${ }^{l}$, Gu JIAN ${ }^{l}$, Li HONGSHENG ${ }^{1, *}$, Armghan SHAHZAD ${ }^{1,3}$, \\ Muhammad ASIM ${ }^{1,3}$, Zhao HONG ${ }^{l}$, Yang MUJUN ${ }^{l}$ \\ ${ }^{1}$ Institute of Food Crops, Yunnan Academy of Agricultural Sciences, Kunming, CHINA \\ ${ }^{2}$ Field Crops Research Institute, Agricultural Research Center, EGYPT \\ ${ }^{3}$ Pakistan Agricultural Research Council, Islamabad, PAKISTAN \\ + First and second authors contributed equally in this manuscript \\ *Corresponding author: lhsyaas@163.com
}

Received: 10.12.2018

\begin{abstract}
This study was conducted to identify desirable wheat germplasm for superior hybrid cross combinations through 1) identifying the best parents with high combining ability (CA) and 2) estimating heterosis effects. Two thermophoto-sensitive-genic-male-sterile lines (K456s and K78s) as female parents and 60 restorer lines as males were crossed according to line $\times$ tester hybridization method. The observed variation for most of the nine studied characters and $<1$ ratio for general (G)/specific (S) CA suggested governance of non-additive gene action. The GCA for K456s was higher than K78s in six out of nine characters, while restorers $2016 \mathrm{Y}_{2}-2776$ and $2016 \mathrm{Y}_{2}$ 4117 had the highest in five characters. The crosses K78s/R31 and K456s/R2 had highest positive SCA values in five and six characters, respectively. The crosses $\mathrm{K} 78 \mathrm{~s} / \mathrm{R43}$ and $\mathrm{K456s} / \mathrm{R} 21$ had the overall highest significant positive heterosis estimates for the best cross with respect to yield plant ${ }^{-1}$ and has the potential for utilization in hybrid wheat breeding program.
\end{abstract}

Keywords: Combining ability, heterosis, hybrid wheat, TPSGMS, traits improvement

\section{INTRODUCTION}

Wheat is the most widely grown and consumed cereal food crop in the world. A current annual production level is more than 651 million tons on a total production area of 217 million hectares. By the year 2050, the world population is estimated to be 9 billion and the demand for wheat will exceed 900 million tons (Tadesse et al., 2013). Hybrid wheat is being recognized as a preferred approach to improve wheat yield. The application of hybrid wheat will greatly increase the food production and make significant contribution to food security in the wake of population increase, climate change associated threats and diminishing natural resources. During 2009 to 2012, the new wheat hybrids were grown on about 66,700 hectares for demonstration with the average yield increase of 15.7 $\%$ in 11 provinces in China (Zhao, 2013).

Male sterility is an essential trait in hybrid seed production for self-pollinated crops like wheat. The use of cytoplasmic male sterile lines (CMS) or thermo photo sensitive genic male sterile (TPSGMS) line as female parent are preferred in wheat hybrid seed production. The TPSGMS lines are characterized by low-temperature and short-day induced sterility, while high-temperature and long-day induced fertility. The two line system using TPSGMS lines does not need maintainer lines and a wider restorer source. Moreover, it has a simple seed production procedure (Song et al., 2005).

Although the wheat hybrids with $>15 \%$ yield increase have been developed (Anonymous, 2015), accelerating yield improvements with stability and stress resistance with wider adaptability under varying agro-ecologies is still the key issue to be solved for the large-area application of hybrid wheat.

The knowledge of combining ability (CA) influencing yield and its components is useful to assess differences among the genotypes and also, elucidate the nature and magnitude of gene actions involved (Salgotra et al., 2009; Fasahat et al., 2016). Information of general and specific combining abilities influencing yield and its components has become increasingly important for plant breeders to select appropriate parents while developing hybrids (Rasheda et al., 2014). Line $\times$ tester analysis is one of the most powerful tools for predicting the general combining ability (GCA) of parents and selecting suitable parents and crosses with high specific combining ability (SCA) (Rashid et al., 2007; Saeed et al., 2001; Krystkowiak et al., 2008; 
Jain and Sastry, 2012). Thus, the main goal of hybrid breeding is to systematically exploit heterosis. Heterosis of a hybrid is expected to increase with the genetic divergence between its parents (Melchinger, 1999). This study, thus, aimed to identify desirable wheat germplasm for superior hybrid cross combinations through 1) identifying the best parents with high combining ability (CA) and 2) estimating heterosis effects.

\section{MATERIALS AND METHODS}

Plant materials

Two TPSGMS lines (K78S and K456S), 60 restorer lines (R1 to R56 from Food Crops Research Institute and R57 to R60 from Mexico), two local checks (Yun mai 53 and Yun mai 56), and their 120 hybrid combinations were used in this study (Table 1).

Table 1. Parent materials used for this study

\begin{tabular}{|c|c|c|c|c|c|}
\hline Ser \# & Parent & Ser \# & Parent & Ser \# & Parent \\
\hline $\mathrm{S} 1$ & K78s & R20 & $2016 Y_{2}-3972$ & R41 & $2016 Y_{2}-4123$ \\
\hline $\mathrm{S} 2$ & $\mathrm{~K} 456 \mathrm{~s}$ & $\mathrm{R} 21$ & $2016 Y_{2}-3988$ & $\mathrm{R} 42$ & $2016 Y_{2}-4124$ \\
\hline $\mathrm{R} 1$ & $2016 Y_{2}-1987$ & R22 & $2016 Y_{2}-3989$ & $\mathrm{R} 43$ & $2016 Y_{2}-4132$ \\
\hline $\mathrm{R} 2$ & $2016 Y_{2}-2301$ & R23 & $2016 Y_{2}-4001$ & $\mathrm{R} 44$ & $2016 Y_{2}-4134$ \\
\hline R3 & $2016 Y_{2}-2652$ & R24 & $2016 Y_{2}-4022$ & $\mathrm{R} 45$ & $2016 Y_{2}-4181$ \\
\hline $\mathrm{R} 4$ & $2016 Y_{2}-2776$ & $\mathrm{R} 25$ & $2016 Y_{2}-4032$ & $\mathrm{R} 46$ & $2016 Y_{2}-4183$ \\
\hline R5 & $2016 Y_{2}-3613$ & R26 & $2016 Y_{2}-4033$ & $\mathrm{R} 47$ & $2016 Y_{2}-4184$ \\
\hline R6 & $2016 Y_{2}-3766$ & R27 & $2016 Y_{2}-4052$ & $\mathrm{R} 48$ & $2016 Y_{2}-4197$ \\
\hline R7 & $2016 Y_{2}-3768$ & R28 & $2016 Y_{2}-4078$ & R49 & $2016 Y_{2}-4201$ \\
\hline $\mathrm{R} 8$ & $2016 Y_{2}-3874$ & R29 & $2016 Y_{2}-4090$ & R50 & $2016 Y_{2}-4203$ \\
\hline R9 & $2016 Y_{2}-3876$ & R30 & $2016 Y_{2}-4094$ & R51 & $2016 Y_{2}-4205$ \\
\hline $\mathrm{R} 10$ & $2016 Y_{2}-3883$ & R31 & $2016 Y_{2}-4097$ & R52 & $2016 Y_{2}-4208$ \\
\hline R11 & $2016 Y_{2}-3884$ & R32 & $2016 Y_{2}-4098$ & R53 & $2016 Y_{2}-4217$ \\
\hline $\mathrm{R} 12$ & $2016 Y_{2}-3889$ & R33 & $2016 Y_{2}-4102$ & R54 & $2016 Y_{2}-4221$ \\
\hline $\mathrm{R} 13$ & $2016 Y_{2}-3901$ & R34 & $2016 Y_{2}-4106$ & R55 & $2016 Y_{2}-4223$ \\
\hline $\mathrm{R} 14$ & $2016 Y_{2}-3902$ & R35 & $2016 Y_{2}-4108$ & R56 & $2016 Y_{2}-4224$ \\
\hline $\mathrm{R} 15$ & $2016 Y_{2}-3907$ & $\mathrm{R} 36$ & $2016 Y_{2}-4111$ & R57 & 2016CIM-1101 \\
\hline R16 & $2016 Y_{2}-3913$ & R37 & $2016 Y_{2}-4117$ & R58 & 2016CIM-2012 \\
\hline $\mathrm{R} 17$ & $2016 Y_{2}-3917$ & R38 & $2016 Y_{2}-4118$ & R59 & 2016CIM-6152 \\
\hline $\mathrm{R} 18$ & $2016 Y_{2}-3919$ & R39 & $2016 Y_{2}-4119$ & R60 & 2016CIM-6203 \\
\hline $\mathrm{R} 19$ & $2016 Y_{2}-3962$ & $\mathrm{R} 40$ & $2016 Y_{2}-4122$ & $\mathrm{CK}$ & $\begin{array}{c}\text { Yun Mai } 53 \text { and } \\
\text { Yun Mai } 56\end{array}$ \\
\hline
\end{tabular}

\section{Field experiment}

The present investigation was carried out at experimental field of Songming Agricultural Research Station, Yunnan Academy of Agricultural Sciences $\left(25.4043^{\circ} \mathrm{N}, 103.625^{\circ} \mathrm{E}, 1872 \mathrm{~m}\right.$ above sea level) during 2016 and 2017 growing seasons. In March 2016, two TPSGMS lines as female and 60 restorer lines as male were sown to produce 120 hybrid wheat crosses according to the $2 \times 60$ line $\times$ tester hybridization method. In October the parents (male, female and checks) and $120 \mathrm{~F}_{1}$ crosses were sown in the field. The parents and $F_{1} s$ were evaluated in a randomized complete block design in triplicate. Each genotype was grown in single row, $2 \mathrm{~m}$ long and $23.33 \mathrm{~cm}$ apart, with plant spacing of $15 \mathrm{~cm}$. The experiment was surrounded by a protective row. The recommended package for Daejeon area was followed for cultural practices. At maturity stage, data were recorded on three randomly selected plants per row in each of the three replications.

\section{Data recording}

Data of nine biometrical traits including yield plant ${ }^{-}$ ${ }^{1}(\mathrm{Y} / \mathrm{P})$, number of spikes plant $^{-1}(\mathrm{~S} / \mathrm{P})$, thousand grain weight (TGW), number of grains $\operatorname{spike}^{-1}(\mathrm{G} / \mathrm{S})$, spike length
(SL), spikelets numbers (SN), fertile spikelets number (FSN), sterile spikelets number (SSN) and plant height (PHt) was recorded. The Y/P of sterile lines is the yield of outcrossing.

\section{Statistical analysis}

All studied characters were statistically analyzed on plot mean basis according to Steel and Torrie (1980) to test the significance of 182 different genotypes. Mean squares for genotypes (parents and $F_{1} s$ ) were partitioned among parents, $F_{1}$ crosses and parents vs crosses (Kearsey and Pooni, 1996). Treatments were compared using the LSD values (Gomez and Gomez, 1984). Line $\times$ tester analysis was performed for all the studied characters. CA and gene effects were studied following Singh and Chaudhary (1985). The $t$-test was used to test whether CA effects were different from 0 . These analyses were done using Excel and Agrobase 99 computer program (Anonymous, 1999)

\section{The estimates of heterosis}

Generally, the expression of increased vigor of the $F_{1}$ hybrid over its parents is called heterosis as proposed by Mather (1949) and Mather and Jinks (1982). However, 
following three approaches are usually used for estimation of heterosis:

1- Mid-parent heterosis or heterosis over the mean parents (MP). The amount of heterosis as proposed by Mather (1949) and Mather and Jinks (1982) was determined as the increase of the $\mathrm{F}_{1}$ hybrid mean over the average of its two parents as follows:

$$
\%(\mathrm{MP})=\left[\left(\mathrm{F}_{1}-\mathrm{MP}\right) / \mathrm{MP}\right] \times 100 \text {. }
$$

2- Heterobeltiosis or heterosis over the better parent (BP). The heterosis of an individual cross was determined as the increase of the $F_{1}$ hybrid mean over its better parent, as follows:

$$
\%(\mathrm{BP})=\left[\left(\mathrm{F}_{1}-\mathrm{BP}\right) / \mathrm{BP}\right] \times 100 .
$$

3- Standard heterosis or heterosis over the best check cultivar $(\mathrm{SH})$. The percent increase or decrease of $F_{1}$ hybrids over best check was calculated to estimate possible heterotic effects for the above mentioned parameters (Fonseca and Patterson, 1968) as follows:

$$
\%(\mathrm{SH})=\left[\left(\mathrm{F}_{1}-\mathrm{SH}\right) / \mathrm{SH}\right] \times 100 .
$$

LSD values were calculated to test the significance of the heterosis effects over MP, BP and check cultivar, according to the formula that was suggested by Wynne et al. (1970).

\section{RESULTS and DISCUSSION}

Analyses of variance

The highly significant mean sum of squares (SS) due to genotypes (parents and hybrids) for all studied characters in this investigation (Table 2) and non-significant for replication indicated the existence of sufficient variation in the studied material (Fisher and Yates, 1967). These highly significant differences in all characters, indicating that the parents possessed alleles with different additive effects. Significant differences were noticed among the progenies, revealing that the parents were diverse for the studied characters and that this diversity could be transmitted to the offspring. A comparison of the parents versus $F_{1}$ crosses revealed significant differences for all characters except $\mathrm{S} / \mathrm{P}$, reflecting a sort of heterosis for these characters. This showed that there was significant variation among lines, testers and hybrids, hence it is possible to calculate the GCA and SCA in the populations. The $<1$ ratio of GCA to SCA variances for all studied characters indicated governance of predominantly non-additive gene action for these characters.

\begin{tabular}{|c|c|c|c|c|c|c|c|c|c|c|}
\hline SoV & df & $\mathbf{Y} / \mathbf{P}$ & $\mathbf{S} / \mathbf{P}$ & TGW & G/S & SL & SN & FSN & SSN & PHt \\
\hline $\mathbf{R}$ & 2 & 39.75 & 3.505 & 38.58 & 36.62 & $2.57 *$ & $5.85^{*}$ & 2.41 & $1.34 *$ & 28.69 \\
\hline $\mathbf{G}$ & 181 & $34.63 * *$ & $4.70 * *$ & $150.74 * *$ & $236.96 * *$ & $3.95 * *$ & $6.25 * *$ & $6.71 * *$ & $1.14 * *$ & $136.8 * *$ \\
\hline $\mathbf{P}$ & 61 & $32.11 * *$ & $4.27 * *$ & $185.19^{* *}$ & $256.91 * *$ & $6.88^{* *}$ & $8.19 * *$ & $9.19 * *$ & $1.03 * *$ & $175.9^{* *}$ \\
\hline C & 119 & $35.96 * *$ & $4.97 * *$ & $126.26^{* *}$ & $190.13 * *$ & $2.44^{* *}$ & $4.54 * *$ & $5.38 * *$ & $0.86^{*}$ & $115.5^{* *}$ \\
\hline PvC & 1 & $28.64 *$ & 1.75 & $961.15^{* *}$ & $4591.9 * *$ & $5.41 * *$ & $91.65 * *$ & $12.98 * *$ & $40.80 * *$ & $293.8 * *$ \\
\hline $\mathbf{L}$ & 1 & $100.59 *$ & $18.87 * *$ & 42.38 & $3858.7 * *$ & $3.85^{*}$ & $162.6 * *$ & $209.4 * *$ & $2.95 * *$ & 13.23 \\
\hline $\mathbf{T}$ & 59 & $45.53 * *$ & $6.28 * *$ & $176.60 * *$ & $221.21 * *$ & $3.64 * *$ & $4.47 * *$ & $5.08 * *$ & $1.17 * *$ & $195.2 * *$ \\
\hline LxT & 59 & $25.31 *$ & $3.42 *$ & $77.35^{* *}$ & $96.88^{* *}$ & $1.21 * *$ & 1.92 & 2.221 & $0.51 *$ & $37.49 * *$ \\
\hline $\mathbf{R}$ & 362 & 16.61 & 2.666 & 37.38 & 56.714 & 0.678 & 1.508 & 1.898 & 0.293 & 13.217 \\
\hline V-GC & & 0.04 & 0.01 & 0.18 & 0.35 & 0.01 & 0.01 & 0.01 & 0.00 & 0.29 \\
\hline $\mathbf{V - S C}$ & & 2.90 & 0.25 & 13.32 & 13.39 & 0.18 & 0.14 & 0.11 & 0.07 & 8.09 \\
\hline \multicolumn{2}{|c|}{$\begin{array}{l}\text { GCA/SCA var. } \\
\text { ratio }\end{array}$} & 0.01 & 0.02 & 0.01 & 0.03 & 0.03 & 0.07 & 0.11 & 0.01 & 0.04 \\
\hline
\end{tabular}

Table 2. Analysis of variance of 9 agronomic characters for genotypes, testers, lines and 120 hybrid wheat combinations and Line $\mathrm{x}$ Tester analysis.

Note: "*" and "**"represent significant differences at $\mathrm{P}<5 \%$ and $\mathrm{P}<1 \%$ levels, respectively. For characters Y/P represents Yield Plant ${ }^{-1}, \mathrm{~S} / \mathrm{P}$ Spikes Plant $^{-1}$, TGW Thousand Grain Weight, G/S Grains Spike ${ }^{-1}$, SL Spike Length, SN Spikelets Number, FSN Fertile Spikelets Number, SSN Sterile Spikelets Number, PHt Plant Height. SoV stands for Sources of Variation, R Replication, G Genotypes, P Parents, C Crosses, PvC Parents vs Crosses, L Lines, T Testers, LxT Line x Tester, R Residual

\section{Analyses of combining ability}

$\mathrm{CA}$ is an estimation of the value of genotypes on the basis of their offspring performance in some definite mating design (Allard, 1960). Average performance of parental line in series of cross combinations is generally referred to as GCA and is mainly attributed to additive and additivexadditive gene effects. The GCA effect values of 9 agronomic traits of 120 hybrid wheat parents (Table 3 ) indicated that the third generation of wheat temperature and photosensitive sterile line K456s had GCA higher than K78s sterile line with respect to $\mathrm{Y} / \mathrm{P}, \mathrm{TGW}, \mathrm{G} / \mathrm{S}, \mathrm{SL}, \mathrm{SN}$ and FSN. K78s sterile line had the higher GCA of S/P, SSN and PHt. Three restorer lines recorded positive significant GCA effects for $\mathrm{Y} / \mathrm{P}$, among which $2016 \mathrm{Y}_{2}-2776$ (R4) recorded the highest positive significant GCA effect (5.70) followed by $2016 \mathrm{Y}_{2}-4132$ (R43) (5.06), and $2016 \mathrm{Y}_{2}-3919$ (R18) (4.70) indicating that they were good general combiners for Y/P. 2016Y ${ }_{2}-4224$ (R56), 2016Y ${ }_{2-4117}$ (R37) and 2016CIM-1101 (R57) recorded the lowest negative GCA for Y/P. Four restorer lines recorded positive significant GCA effects for $\mathrm{S} / \mathrm{P}$, among which $2016 Y_{2}-3884$ (R11) recorded the highest positive significant GCA effect (2.47) followed by $2016 Y_{2}-3919$ 
(R18) (2.43), 2016Y 2 -2776 (R4) (1.98) and (R10) (1.76) indicating that they were good general combiners for S/P. 2016Y ${ }_{2}-4217$ (R53) and 2016 $\mathrm{Y}_{2}-4224$ (R56) recorded the lowest negative GCA for S/P.

Six restorer lines recorded positive significant GCA effects for TGW, indicating being good general combiners for this character. Among them, 2016Y $\mathrm{Y}_{2}-4118$ (R38) recorded the highest GCA effect (12.3) followed by $2016 \mathrm{Y}_{2}-3889$ (R12) (8.86), 2016 $\mathrm{Y}_{2}-4217$ (R53) (8.38),

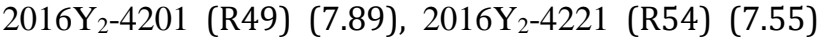

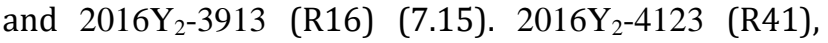
$2016 \mathrm{Y}_{2}-3766$ (R6), 2016 $\mathrm{Y}_{2}-3768$ (R7) and 2016Y $\mathrm{Y}_{2}-3613$ (R5) recorded the lowest negative GCA.

For G/S, five restorer lines were good general combiners and recorded positive significant GCA effects, among them 2016Y 2 -3972 (R20) recorded the highest positive significant GCA effect (13.9) followed by $2016 \mathrm{Y}_{2}-4181$ (R45) (10.2), 2016 $\mathrm{Y}_{2}-4117$ (R37) (9.09), 2016 $\mathrm{Y}_{2}-4090$ (R29) (8.5) and 2016 $\mathrm{Y}_{2}-2776$ (R4) (8.19). 2016Y ${ }_{2}-3913$ (R16), 2016Y ${ }_{2}-4078$ (R28) and 2016CIM6203 (R60) recorded the lowest negative GCA for G/S.

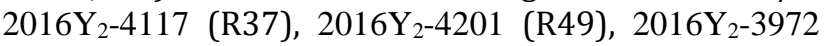
(R20), 2016Y $2-4022$ (R24) and 2016Y $\mathrm{Y}_{2}-4217$ (R53) recorded positive significant GCA for SL, while $2016 \mathrm{Y}_{2-}$ 3919 (R18), 2016 ${ }_{2}-4078$ (R28) and 2016 ${ }_{2}-3988$ (R21) recorded the lowest negative GCA. The highest positive GCA for SN recorded by $2016 \mathrm{Y}_{2}-4117$ (R37), 2016Y ${ }_{2-}$ 3913 (R16) and 2016Y $2-4183$ (R46), while 2016CIM6152 (R59) and 2016CIM-2012 (R58) recorded the lowest negative GCA. The highest positive GCA for FSN recorded by 2016 $\mathrm{Y}_{2}-4117$ (R37), 2016 ${ }_{2}-3972$ (R20) and 2016Y ${ }_{2}-4197$ (R48), while 2016CIM-2012 (R58) recorded the lowest negative GCA. 2016Y $2-3972$ (R20), 2016Y ${ }_{2}-4197$ (R48), 2016 ${ }_{2}-4201$ (R49), 2016Y ${ }_{2}-4117$ (R37) and 2016Y $\mathrm{Y}_{2}-2776$ (R4) recorded the lowest negative GCA for SSN. 13 restorer lines recorded positive and significant GCA effects for PHt. We can conclude that $2016 \mathrm{Y}_{2}-2776$ (R4) had the highest general combining ability in five characters $(\mathrm{Y} / \mathrm{P}, \mathrm{S} / \mathrm{P}, \mathrm{G} / \mathrm{S}, \mathrm{SSN}$ and PHt). 2016 $\mathrm{Y}_{2}-4117$ (R37) had the highest general combining ability in five characters (G/S, SL, SN, FSN and SSN).

Deviation in the performance of a cross expected on the basis of average performance of parental lines is mainly attributed to dominant and epistatic effects and is termed as specific combining ability i.e., non-additive part. Nonadditive component is presence for the controlling traits necessitates exploitation of hybrid vigor in wheat breeding scheme.
The SCA effects of 120 hybrids for all studied characters were estimated and the highest $5 \%$ values for each character were presented in Tables 4 . The best specific crosses for $\mathrm{Y} / \mathrm{P}$ with first sterile line were $\mathrm{K} 78 \mathrm{~s} / \mathrm{R} 43$, $\mathrm{K} 78 \mathrm{~s} / \mathrm{R} 31, \mathrm{~K} 78 \mathrm{~s} / \mathrm{R} 35, \mathrm{~K} 78 \mathrm{~s} / \mathrm{R} 4, \mathrm{~K} 78 \mathrm{~s} / \mathrm{R} 8$ and K78s/R46. The best specific crosses for $\mathrm{Y} / \mathrm{P}$ with second sterile line were K456s/R21，K456s/R17，K456s/R14，K456s/R2, $\mathrm{K} 456 \mathrm{~s} / \mathrm{R} 20$ and $\mathrm{K} 456 \mathrm{~s} / \mathrm{R} 32$. Non-significant positive and negative SCA values were recorded for S/P and SSN. The best specific crosses for TGW with first sterile line were $\mathrm{K} 78 \mathrm{~s} / \mathrm{R} 31, \mathrm{~K} 78 \mathrm{~s} / \mathrm{R} 37 ， \mathrm{~K} 78 \mathrm{~s} / \mathrm{R} 53, \mathrm{~K} 78 \mathrm{~s} / \mathrm{R} 42 ， \mathrm{~K} 78 \mathrm{~s} / \mathrm{R} 35$ and $\mathrm{K} 78 \mathrm{~s} / \mathrm{R} 50$, while with second sterile line were K456s/R51， K456s/R36， K456s/R27， K456s/R16, $\mathrm{K} 456 \mathrm{~s} / \mathrm{R} 32$ and K456s/R57. The crosses K78s/R24 and K78s/R31 had positive and significant SCA values for SL. The crosses K78s/R57 and K456s/R16 had positive and significant SCA values for SN and FSN. Positive and significant SCA values for PHt were recorded by K78s/R35 and $\mathrm{K} 456 \mathrm{~s} / \mathrm{R} 14$. The cross $\mathrm{K} 78 \mathrm{~s} / \mathrm{R} 31$ had highest positive values in five characters, while $\mathrm{K} 78 \mathrm{~s} / \mathrm{R} 35, \mathrm{~K} 78 \mathrm{~s} / \mathrm{R} 24$, $\mathrm{K} 78 \mathrm{~s} / \mathrm{R} 57$ and $\mathrm{K} 78 \mathrm{~s} / \mathrm{R} 53$ crosses had highest positive values in three characters. K456s/R2 cross had highest positive values in six characters, while K456s/R17 and K456s/R14 had highest positive values in four characters. This information could be used for estimating and selecting superior cross combinations while exploiting heterosis and selecting homozygous lines in wheat, which is a self pollinated plant (Kose, 2017; Istipliler et al., 2015)

\section{Estimates of heterosis effects}

Many researchers have emphasized the utilization of heterosis percent as an important criterion for evaluation of hybrids. Therefore, the knowledge about the magnitude of heterosis would help in selection of best cross combination. Heterosis for $\mathrm{Y} / \mathrm{P}, \mathrm{S} / \mathrm{P}, \mathrm{TGW}$ and $\mathrm{G} / \mathrm{S}$ was estimated and presented in Table 5. The highest combination for $\mathrm{Y} / \mathrm{P}$ of heterosis over BP, MP and SH was K78s/R4, K456s/R20 and $\mathrm{K} 456 \mathrm{~s} / \mathrm{R} 21 . \mathrm{K} 78 \mathrm{~s} / \mathrm{R} 43$ and $\mathrm{K} 78 \mathrm{~s} / \mathrm{R} 18$ combinations recorded high positive heterosis over MP and SH for Y/P. The combinations K78s/R37, K78s/R27, K78s/R56, $\mathrm{K} 456 \mathrm{~s} / \mathrm{R} 31, \mathrm{~K} 456 \mathrm{~s} / \mathrm{R} 37$, and $\mathrm{K} 456 \mathrm{~s} / \mathrm{R} 55$ recorded lowest negative heterosis over MP and SH.

For $\mathrm{S} / \mathrm{P}$, heterosis were significant and positive in $\mathrm{K} 78 \mathrm{~s} / \mathrm{R} 11, \mathrm{~K} 78 \mathrm{~s} / \mathrm{R} 43, \mathrm{~K} 78 \mathrm{~s} / \mathrm{R} 34$ crosses over BP and in K78s/R38, K78s/R48, K78s/R43, K456s/R59, K456s/R25, and K456s/R43 crosses over MP. The combinations K78s/R53, K78s/R37, K78s/R56, K456s/R55, K456s/R39 and $\mathrm{K} 456 \mathrm{~s} / \mathrm{R} 56$ recorded lowest negative heterosis for S/P. 
Table 3. General combining ability value of 9 agronomic traits of sterile and restorer lines of hybrid wheat

\begin{tabular}{|c|c|c|c|c|c|c|c|c|c|}
\hline Parents & $\mathbf{Y} / \mathbf{P}$ & $\mathbf{S} / \mathbf{P}$ & TGW & G/S & SL & SN & FSN & SSN & PHt \\
\hline K78s & -0.5286 & 0.2289 & -0.3431 & -3.2739 & -0.1035 & -0.6722 & -0.7627 & 0.0905 & 0.1917 \\
\hline K456s & 0.5286 & -0.2289 & 0.3431 & $3.27 * *$ & 0.1035 & $0.67 * *$ & $0.76^{* *}$ & -0.0905 & -0.1917 \\
\hline $\mathrm{R} 1$ & -3.3037 & -0.9345 & -4.9208 & -2.4367 & 0.0552 & 0.3906 & 0.2668 & 0.1239 & -5.725 \\
\hline $\mathrm{R} 2$ & 1.5213 & 0.3711 & -2.7173 & 3.4191 & 0.1861 & -0.7493 & -0.69 & -0.0593 & -1.0583 \\
\hline $\mathrm{R} 3$ & 3.8322 & 0.899 & 4.6413 & 1.5725 & 0.0656 & -0.4775 & 0.0834 & -0.561 & $8.11 * *$ \\
\hline $\mathrm{R} 4$ & $5.70 * *$ & $1.98 *$ & -4.0842 & $8.19^{*}$ & 0.0103 & 0.3942 & 0.9944 & $-0.60 *$ & $9.44 * *$ \\
\hline R5 & -0.3698 & 0.6766 & -7.5098 & 0.9762 & -0.1045 & 0.4203 & 0.5337 & -0.1133 & $4.78 *$ \\
\hline R6 & 0.9752 & 1.2322 & -11.8094 & 7.4619 & 0.392 & 0.6216 & 1.1528 & -0.5312 & $4.78^{*}$ \\
\hline R7 & -2.5006 & -0.4623 & -11.8425 & 6.5232 & 0.4026 & 0.2012 & 0.3816 & -0.1804 & $5.44 * *$ \\
\hline R8 & 2.3397 & 1.0933 & 1.3639 & -0.7571 & 0.2315 & 0.131 & -0.0292 & 0.1602 & 1.9417 \\
\hline R9 & -0.4251 & 0.4822 & -3.364 & -2.0442 & 0.5251 & 1.152 & 1.2038 & -0.0518 & -0.0583 \\
\hline $\mathrm{R} 10$ & 1.9319 & $1.76^{*}$ & 1.7222 & -7.3746 & -0.2845 & 0.3523 & -0.2692 & 0.6215 & -3.225 \\
\hline R11 & 2.848 & $2.47 * *$ & -6.5294 & 0.3103 & 0.5909 & 1.1412 & 1.0439 & 0.0973 & -3.225 \\
\hline R12 & 4.4369 & 0.9266 & $8.86^{* *}$ & -0.8854 & -0.0815 & -1.0295 & -1.4726 & 0.4431 & 3.275 \\
\hline R13 & -2.8837 & -0.6845 & -2.1615 & -4.8277 & 0.8413 & 0.6403 & -0.0504 & 0.6906 & -9.225 \\
\hline R14 & 0.3669 & -0.1845 & 3.0339 & -1.6096 & -0.9689 & -0.2048 & -0.2023 & -0.0026 & -5.5583 \\
\hline $\mathrm{R} 15$ & -4.1184 & -1.1428 & -1.9217 & -4.6839 & 0.4683 & -0.6633 & -0.0756 & -0.5877 & -7.3917 \\
\hline R16 & -2.9776 & -0.5178 & $7.15^{*}$ & -14.0678 & 0.545 & $2.26 * *$ & 1.4015 & 0.8626 & -0.225 \\
\hline $\mathrm{R} 17$ & -0.4887 & -0.3095 & 2.5604 & -5.31 & -0.8967 & -1.4378 & -0.895 & -0.5429 & 0.4417 \\
\hline $\mathrm{R} 18$ & $4.70 * *$ & $2.43 * *$ & -0.965 & -1.7035 & -1.1604 & -1.1575 & -0.9894 & -0.1681 & -15.0583 \\
\hline R19 & -2.1198 & -0.8789 & -6.907 & 2.8834 & -0.6582 & -0.2522 & -0.6933 & 0.4411 & -5.225 \\
\hline $\mathrm{R} 20$ & 2.5449 & -0.365 & -1.9065 & $13.95 * *$ & $1.21 * *$ & 1.1352 & $2.16^{* *}$ & $-1.03 * *$ & -7.3917 \\
\hline $\mathrm{R} 21$ & 3.6363 & 0.4266 & 3.0019 & 4.215 & -1.9388 & -1.2421 & -1.3034 & 0.0613 & -5.5583 \\
\hline $\mathrm{R} 22$ & 0.7227 & -0.6012 & 2.333 & 4.8208 & -0.7646 & -0.4777 & -0.5691 & 0.0913 & -1.5583 \\
\hline $\mathrm{R} 23$ & -2.797 & -1.1012 & -1.6086 & -1.0197 & 0.4958 & 0.0608 & 0.3672 & -0.3064 & -2.725 \\
\hline $\mathrm{R} 24$ & 0.4524 & 0.0377 & -3.9886 & 4.4848 & $0.998 *$ & 0.367 & 0.299 & 0.0681 & -6.3917 \\
\hline $\mathrm{R} 25$ & 1.813 & 1.3988 & -3.3133 & -1.8385 & -0.3727 & 0.2145 & -0.0336 & 0.2481 & -5.225 \\
\hline $\mathrm{R} 26$ & -2.3387 & -0.1567 & -3.9429 & -5.2037 & -0.5545 & 0.0407 & -0.3643 & 0.405 & -6.8917 \\
\hline $\mathrm{R} 27$ & -3.3592 & -0.3234 & -4.9456 & -7.3473 & -0.8465 & -0.0691 & -0.1614 & 0.0923 & -5.0583 \\
\hline $\mathrm{R} 28$ & -2.3801 & 0.7044 & -3.3226 & -12.2625 & -1.3955 & -0.9984 & -1.3723 & 0.3739 & -2.225 \\
\hline $\mathrm{R} 29$ & 0.7244 & 0.6766 & 1.4559 & $8.51 *$ & -0.5578 & -0.0461 & -0.2905 & 0.2444 & $3.94 *$ \\
\hline $\mathrm{R} 30$ & -0.7192 & 0.0933 & -4.6442 & -1.6477 & 0.0945 & -0.3237 & 0.0276 & -0.3513 & -4.5583 \\
\hline R31 & -1.2748 & -0.5178 & -2.4588 & 0.9928 & -0.7917 & -0.6823 & -0.1645 & -0.5178 & -0.0583 \\
\hline $\mathrm{R} 32$ & -1.1376 & -1.0317 & $6.6^{* *}$ & -3.8389 & 0.481 & -0.6228 & -0.8247 & 0.2019 & $10.61 * *$ \\
\hline R33 & -1.3628 & -0.7956 & $7.79 *$ & -6.6264 & -0.8829 & -1.0632 & -1.0617 & -0.0016 & 1.9417 \\
\hline R34 & 4.2088 & 1.7183 & 0.9979 & 3.9896 & -0.945 & -0.4497 & -0.2106 & -0.2391 & -3.8917 \\
\hline $\mathrm{R} 35$ & -1.0748 & -0.1567 & -6.3638 & 3.6579 & 0.3298 & 1.1654 & 1.0479 & 0.1174 & 1.4417 \\
\hline R36 & 0.9224 & -0.1012 & 3.0403 & 0.4529 & -0.5489 & -0.3308 & -1.0514 & 0.7206 & -2.5583 \\
\hline R37 & -5.0706 & -1.7956 & -11.4585 & $9.09 * *$ & $2.50 * *$ & $2.39 * *$ & $3.05 * *$ & $-0.68^{*}$ & -5.8917 \\
\hline R38 & 4.0924 & -0.3512 & $12.30 * *$ & 5.6711 & 0.5943 & 1.2764 & 0.9751 & 0.3013 & $7.94 * *$ \\
\hline R39 & -1.6764 & -1.3512 & 1.2537 & 4.2209 & 0.4731 & 0.3367 & -0.1429 & 0.4796 & $4.94 *$ \\
\hline $\mathrm{R} 40$ & 1.2952 & -0.1845 & 6.2258 & 0.6153 & -0.9179 & 0.6194 & 0.4878 & 0.1316 & $15.11 * *$ \\
\hline R41 & 3.0445 & -0.4206 & 9.4589 & 5.2223 & 0.1549 & -0.4551 & -0.4646 & 0.0094 & -0.5583 \\
\hline $\mathrm{R} 42$ & -1.3312 & 0.1488 & -0.1348 & -4.6855 & -0.0644 & 1.0073 & 0.8787 & 0.1286 & 0.775 \\
\hline R43 & $5.06^{* *}$ & 1.3155 & 2.5406 & 4.791 & -0.7031 & 0.7128 & 0.7234 & -0.0106 & $8.11 * *$ \\
\hline $\mathrm{R} 44$ & -1.1839 & 0.1211 & 1.0192 & -7.8534 & -0.3456 & -0.1739 & -0.8475 & 0.6737 & $4.78 *$ \\
\hline $\mathrm{R} 45$ & 3.4991 & 0.8155 & -1.7096 & $10.23 * *$ & 0.2393 & -0.1694 & -0.3997 & 0.2303 & 1.275 \\
\hline R46 & 0.1411 & -0.1567 & -4.8847 & 6.9639 & -0.4083 & $1.35^{*}$ & 0.7779 & 0.5678 & -5.5583 \\
\hline R47 & 2.4741 & 0.7044 & -0.0002 & 2.2039 & -0.5278 & -0.0543 & -0.1027 & 0.0484 & -1.8917 \\
\hline R48 & 1.3366 & 0.3988 & 2.849 & 1.9918 & 0.39 & 0.8353 & $1.83^{*}$ & $-0.997 * *$ & -1.0583 \\
\hline R49 & 2.3733 & -0.9067 & $7.89 *$ & $8.56^{*}$ & $1.34 * *$ & 0.1866 & 1.1693 & $-0.98 * *$ & 0.9417 \\
\hline $\mathrm{R} 50$ & 1.2913 & 0.2044 & -0.4267 & 3.5999 & 0.2965 & -0.131 & -0.5856 & 0.4546 & -1.3917 \\
\hline R51 & -1.8417 & -0.8789 & 2.0158 & -1.6746 & -1.1216 & -0.003 & -0.4698 & 0.4668 & -0.5583 \\
\hline R52 & 0.8088 & -0.2123 & 6.2618 & -1.754 & -0.6289 & -0.1843 & -0.487 & 0.3027 & $5.61 *$ \\
\hline R53 & -3.9653 & -2.1289 & $8.38 *$ & 2.8036 & $0.930 *$ & 0.119 & -0.1984 & 0.3174 & 1.4417 \\
\hline R54 & 0.4008 & -0.9067 & $7.55^{*}$ & 2.8274 & 0.8369 & -0.7765 & -0.9585 & 0.182 & -0.3917 \\
\hline R55 & -2.8126 & -1.8512 & 4.1623 & 1.6658 & $1.17 * *$ & -1.1599 & -0.6354 & -0.5245 & -0.0583 \\
\hline R56 & -5.1037 & -1.7956 & 1.0457 & -7.794 & -0.1186 & 0.0178 & 0.2883 & -0.2706 & -2.0583 \\
\hline R57 & -4.7395 & -0.8512 & -3.7601 & -9.5145 & 0.0467 & 0.1136 & 0.0631 & 0.0505 & -3.725 \\
\hline $\mathrm{R} 58$ & -1.3837 & -0.1845 & 4.2151 & -6.8131 & 0.7044 & -1.5765 & -1.5083 & -0.0682 & $7.78 * *$ \\
\hline R59 & -2.0598 & 0.6766 & -6.6736 & -6.9806 & 0.3271 & -1.9141 & -1.2277 & $-0.687 *$ & $6.44 * *$ \\
\hline R60 & -2.6906 & 0.4822 & -1.5355 & -14.2487 & 0.6641 & -0.7754 & -0.4063 & -0.369 & $11.94 * *$ \\
\hline
\end{tabular}

Note: “*” and "** "represent significant differences at $\mathrm{P}<5 \%$ and $\mathrm{P}<1 \%$ levels, respectively. For characters Y/P represents Yield Plant ${ }^{-1}, \mathrm{~S} / \mathrm{P}$ Spikes Plant ${ }^{-1}$, TGW Thousand Grain Weight, G/S Grains Spike ${ }^{-1}$, SL Spike Length, SN Spikelets Number, FSN Fertile Spikelets Number, SSN Sterile Spikelets Number, PHt Plant Height. 
Table 4. The specific combining ability values of the studied traits for top 5\% of 120 hybrid wheat crosses.

\begin{tabular}{|c|c|c|c|c|c|c|c|c|c|}
\hline & $\mathbf{Y} / \mathbf{P}$ & $\mathbf{S} / \mathbf{P}$ & TGW & G/S & SL & SN & FSN & SSN & PH \\
\hline \multirow{12}{*}{ 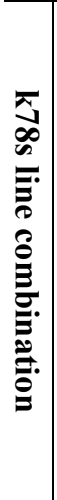 } & K78s/R43 & K78s/R43 & K78s/R31 & K78s/R31 & K78s/R24 & K78s/R57 & K78s/R57 & K78s/R14 & K78s/R35 \\
\hline & $5.236 * *$ & 1.938 & $9.652 * *$ & $13.405 * *$ & $1.437 *$ & $2.588 * *$ & $2.035^{*}$ & 0.742 & 7.1 \\
\hline & K78s/R31 & $\mathrm{K} 78 \mathrm{~s} / \mathrm{R} 8$ & K78s/R37 & K78s/R29 & $\mathrm{K} 78 \mathrm{~s} / \mathrm{R} 31$ & $\mathrm{~K} 78 \mathrm{~s} / \mathrm{R} 24$ & $\mathrm{~K} 78 \mathrm{~s} / \mathrm{R} 24$ & K78s/R37 & $\mathrm{K} 78 \mathrm{~s} / \mathrm{R} 33$ \\
\hline & $4.313 * *$ & 1.493 & $8.550 * *$ & $9.320 * *$ & $1.345^{*}$ & 0.966 & 1.071 & 0.627 & 4.308 \\
\hline & K78s/R35 & K78s/R34 & K78s/R53 & $\mathrm{K} 78 \mathrm{~s} / \mathrm{R} 53$ & $\mathrm{~K} 78 \mathrm{~s} / \mathrm{R} 19$ & $\mathrm{~K} 78 \mathrm{~s} / \mathrm{R} 21$ & $\mathrm{~K} 78 \mathrm{~s} / \mathrm{R} 55$ & K78s/R57 & $\mathrm{K} 78 \mathrm{~s} / \mathrm{R} 53$ \\
\hline & $3.501 * *$ & 1.146 & $7.067 * *$ & $8.191 * *$ & 0.912 & 0.926 & 1.027 & 0.553 & 4.142 \\
\hline & $\mathrm{K} 78 \mathrm{~s} / \mathrm{R} 4$ & K78s/R58 & K78s/R42 & K78s/R55 & $\mathrm{K} 78 \mathrm{~s} / \mathrm{R} 21$ & $\mathrm{~K} 78 \mathrm{~s} / \mathrm{R} 28$ & K78s/R31 & K78s/R34 & $\mathrm{K} 78 \mathrm{~s} / \mathrm{R} 25$ \\
\hline & $2.858 * *$ & 1.104 & $6.960 * *$ & $7.185 * *$ & 0.772 & 0.746 & 0.902 & 0.506 & 3.808 \\
\hline & $\mathrm{K} 78 \mathrm{~s} / \mathrm{R} 8$ & $\mathrm{~K} 78 \mathrm{~s} / \mathrm{R} 48$ & K78s/R35 & K78s/R6 & $\mathrm{K} 78 \mathrm{~s} / \mathrm{R} 16$ & $\mathrm{~K} 78 \mathrm{~s} / \mathrm{R} 18$ & $\mathrm{~K} 78 \mathrm{~s} / \mathrm{R} 18$ & K78s/R19 & $\mathrm{K} 78 \mathrm{~s} / \mathrm{R} 42$ \\
\hline & $2.786 * *$ & 1.077 & $6.112 * *$ & $4.990 * *$ & 0.719 & 0.698 & 0.820 & 0.476 & 3.808 \\
\hline & $\mathrm{K} 78 \mathrm{~s} / \mathrm{R} 46$ & $\mathrm{~K} 78 \mathrm{~s} / \mathrm{R} 12$ & K78s/R50 & K78s/R39 & $78 \mathrm{~s} / \mathrm{R} 53$ & K78s/R55 & K78s/R33 & K78s/R27 & K78s/R9 \\
\hline & $2.378^{*}$ & 0.938 & $3.611 * *$ & $4.265 * *$ & 0.388 & 0.612 & 0.775 & 0.389 & 2.975 \\
\hline \multirow{12}{*}{  } & K456s/R21 & K456s/R2 & K456s & K456s/R48 & K456s/R7 & K456s/R16 & K456s/R16 & K456s/R31 & $\mathrm{K} 456 \mathrm{~s} / \mathrm{R} 14$ \\
\hline & $5.353 * *$ & 1.451 & 5.84 & $8.464 * *$ & 1.094 & $2.100 *$ & $2.225^{*}$ & 0.706 & $5.192 * *$ \\
\hline & K456s/R17 & K456s/R53 & $\mathrm{K} 456 \mathrm{~s} / \mathrm{R} 36$ & K456s/R37 & K456s/R37 & K456s/R35 & $\mathrm{K} 456 \mathrm{~s} / \mathrm{R} 2$ & K456s/R33 & K456s/R22 \\
\hline & $3.967 * *$ & 1.396 & $5.345 * *$ & $7.227 * *$ & 0.981 & 0.748 & 0.980 & 0.505 & 4.8 \\
\hline & K456s/R14 & $\mathrm{K} 456 \mathrm{~s} / \mathrm{R} 24$ & $\mathrm{~K} 456 \mathrm{~s} / \mathrm{R} 27$ & K456s/R21 & $\mathrm{K} 456 \mathrm{~s} / \mathrm{R} 2$ & $\mathrm{~K} 456 \mathrm{~s} / \mathrm{R} 2$ & $\mathrm{~K} 456 \mathrm{~s} / \mathrm{R} 14$ & K456s/R13 & K456s/R34 \\
\hline & $3.584 * *$ & 1.284 & $5.197 * *$ & $7.196 * *$ & 0.665 & 0.741 & 0.958 & 0.465 & \\
\hline & $\mathrm{K} 456 \mathrm{~s} / \mathrm{R} 2$ & $\mathrm{~K} 456 \mathrm{~s} / \mathrm{R} 17$ & $\mathrm{~K} 456 \mathrm{~s} / \mathrm{R} 16$ & K456s/R14 & K456s/R47 & K456s/R32 & K456s/R37 & K456s/R55 & $\mathrm{K} 456 \mathrm{~s} / \mathrm{R} 2$ \\
\hline & $3.550 * *$ & 1.215 & $5.152 * *$ & $6.545 * *$ & & 0.678 & 0.903 & 0.414 & 3.692 \\
\hline & $\mathrm{K} 456 \mathrm{~s} / \mathrm{R} 20$ & $\mathrm{~K} 456 \mathrm{~s} / \mathrm{R} 42$ & $\mathrm{~K} 456 \mathrm{~s} / \mathrm{R} 32$ & K456s / R34 & K456s / R60 & K456s/R41 & $\mathrm{K} 456 \mathrm{~s} / \mathrm{R} 17$ & K456s/R54 & K456s/R31 \\
\hline & $3.013 * *$ & 1.173 & $5.047 * *$ & $5.600 * *$ & 0.508 & 0.585 & 0.669 & 0.389 & 3.692 \\
\hline & K456s/R32 & $\mathrm{K} 456 \mathrm{~s} / \mathrm{R} 30$ & K456s/R57 & K456s / R16 & K456s/R59 & $\mathrm{K} 456 \mathrm{~s} / \mathrm{R} 17$ & $\mathrm{~K} 456 \mathrm{~s} / \mathrm{R} 12$ & K456s/R53 & K456s/R16 \\
\hline & $2.859 * *$ & 1.062 & 4.93 & $5.062 * *$ & 0.476 & 0.576 & 0.584 & 0.356 & 3.192 \\
\hline
\end{tabular}

For TGW, highly significant positive mean heterosis estimates were observed in $\mathrm{SH}$. This showed the tendency of superiority by K456s/R38, K78s/R53, K456s/R16, $\mathrm{K} 78 \mathrm{~s} / \mathrm{R} 52$, K456s/R32 and $\mathrm{K} 78 \mathrm{~s} / \mathrm{R} 54$ in this trait. However, the BP heterosis was significant to highly significant positive in K456s/R12, K456s/R12, K78s/R47, $\mathrm{K} 456 \mathrm{~s} / \mathrm{R} 36, \mathrm{~K} 78 \mathrm{~s} / \mathrm{R} 38$ and $\mathrm{K} 78 \mathrm{~s} / \mathrm{R} 23$. The combination $\mathrm{K} 78 \mathrm{~s} / \mathrm{R} 53$ recorded the highest positive heterosis over MP. The lowest negative heterosis recorded by K456s/R37 over $\mathrm{SH}, \mathrm{BP}$ and MP.

For $\mathrm{G} / \mathrm{S}$, highly significant positive mean heterosis estimates were observed in only two cross (K456s/R20 and $\mathrm{K} 456 \mathrm{~s} / \mathrm{R} 37)$ in $\mathrm{SH}$. However, highest significant positive heterosis recorded by K456s/R20, K456s/R34, K456s/R21 and K78s/R37 over BP. K78s/R29, K78s/R31 and $\mathrm{K} 78 \mathrm{~s} / \mathrm{R} 45$ crosses had the highest significant positive heterosis estimates over MP. We summarized that cross $\mathrm{K} 78 \mathrm{~s} / \mathrm{R} 43$ was the best in $\mathrm{Y} / \mathrm{P}$ and $\mathrm{S} / \mathrm{P}$, however K456s/R21 was the best in $\mathrm{Y} / \mathrm{P}$ and $\mathrm{G} / \mathrm{S}$. This exploitation of heterosis in wheat hybrid development could be of significant economic importance considering the role of wheat in food security around the globe (Hochholdinger and Baldauf, 2018).

\section{Contribution of lines, testers and their interactions towards yield testing}

The proportional contribution of lines, testers and their interactions to total variances for different traits revealed that maximum contribution for all traits was due to tester effect. These lines showed more contribution than line $\mathrm{x}$ tester interaction for SN and FSN (Table 6). 
Table 5. Heterosis estimation using various approaches for yield and yield components

\begin{tabular}{|c|c|c|c|c|c|c|c|c|c|c|c|}
\hline \multicolumn{3}{|c|}{ Entry } & \multicolumn{3}{|c|}{ SH } & \multicolumn{3}{|c|}{ BP } & \multicolumn{3}{|c|}{ MP } \\
\hline \multirow{8}{*}{ 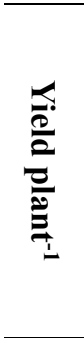 } & \multirow{4}{*}{$\begin{array}{c}\text { Highest } \\
\text { combination }\end{array}$} & \multirow{2}{*}{ K78s } & R43 & R4 & R18 & R6 & R4 & R38 & R43 & R4 & R18 \\
\hline & & & $34.4 * *$ & 23.8 & 7.58 & $126.7 * *$ & $82.2 * *$ & $67.5^{* *}$ & $238.8 * *$ & $212.0 * *$ & $170.9 * *$ \\
\hline & & \multirow{2}{*}{ K456s } & R21 & $\mathrm{R} 20$ & R49 & R21 & R20 & $\mathrm{R} 2$ & $\mathrm{R} 21$ & R20 & R33 \\
\hline & & & $32.9 *$ & 11.9 & 9.8 & $131.4 * *$ & $94.8 * *$ & $89.6^{* *}$ & $165.6^{* *}$ & $116.8 * *$ & $113.4^{*}$ \\
\hline & \multirow{4}{*}{$\begin{array}{c}\text { Lowest } \\
\text { combination }\end{array}$} & \multirow{2}{*}{ K78s } & R37 & $\mathrm{R} 27$ & R56 & $\mathrm{R} 57$ & R60 & R56 & R56 & $\mathrm{R} 27$ & R37 \\
\hline & & & $-60.6 * *$ & $-63.7 * *$ & $-63.9 * *$ & $-60.5^{* *}$ & $-60.1 * *$ & $-57.9 * *$ & -8.9 & -8.4 & -0.7 \\
\hline & & \multirow{2}{*}{$\mathrm{K} 456 \mathrm{~s}$} & $\mathrm{R} 31$ & R37 & R55 & R31 & R37 & $\mathrm{R} 44$ & R31 & R37 & R55 \\
\hline & & & $-56.4 * *$ & $-52.4 * *$ & $-51.7 * *$ & $-52.1 * *$ & $-47.1 * *$ & $-44.5^{* *}$ & -24.1 & -17.1 & -15.8 \\
\hline \multirow{8}{*}{ 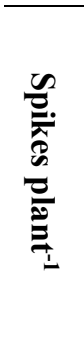 } & \multirow{4}{*}{$\begin{array}{c}\text { Highest } \\
\text { combination }\end{array}$} & \multirow[b]{2}{*}{ K78s } & R11 & R43 & R34 & R11 & R43 & R34 & R38 & R48 & R43 \\
\hline & & & -4.1 & -5.3 & -9.5 & $38.5 * *$ & $36.8 *$ & $30.8 *$ & $112.0 * *$ & $93.9 * *$ & $72.0 * *$ \\
\hline & & \multirow{2}{*}{ K456s } & R18 & $\mathrm{R} 2$ & R47 & $\mathrm{R} 2$ & $\mathrm{R} 47$ & R11 & R59 & R25 & $\mathrm{R} 43$ \\
\hline & & & $-21.9 *$ & $-25.4 *$ & $-27.8^{* *}$ & 18.9 & 15.1 & 12.5 & $157.9 * *$ & 50.7 & 46.4 \\
\hline & \multirow{4}{*}{$\begin{array}{c}\text { Lowest } \\
\text { combination }\end{array}$} & \multirow{2}{*}{ K78s } & R53 & R37 & R56 & R53 & R37 & R56 & R37 & $\mathrm{R} 17$ & R24 \\
\hline & & & $-77.5 * *$ & $-66.9 * *$ & $-62.1 * *$ & $-67.5 * *$ & $-52.1 * *$ & $-45.3 * *$ & -41.7 & $-36.8^{*}$ & -32.5 \\
\hline & & \multirow{2}{*}{$\mathrm{K} 456 \mathrm{~s}$} & R55 & R39 & $\mathrm{R} 56$ & $\mathrm{R} 16$ & $\mathrm{R} 40$ & R3 & R56 & $\mathrm{R} 15$ & $\mathrm{R} 16$ \\
\hline & & & $-63.3 * *$ & $-63.3 * *$ & $-61.0 * *$ & $-50.0 * *$ & $-49.0 * *$ & $-44.4 * *$ & -35.3 & -33.5 & -32.1 \\
\hline \multirow{8}{*}{ 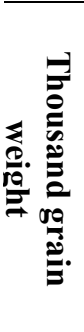 } & \multirow{4}{*}{$\begin{array}{c}\text { Highest } \\
\text { combination }\end{array}$} & \multirow{2}{*}{ K78s } & R53 & R52 & R54 & R47 & R38 & R23 & R53 & R52 & R54 \\
\hline & & & $69.9 * *$ & $54.5 * *$ & $53.9^{* *}$ & $31.4 * *$ & $30.0 * *$ & $29.4 * *$ & $65.7 * *$ & $50.7^{* *}$ & $50.2 * *$ \\
\hline & & \multirow{2}{*}{ K456s } & $\mathrm{R} 38$ & R16 & R32 & $\mathrm{R} 32$ & $\mathrm{R} 12$ & R36 & $\mathrm{R} 12$ & R33 & R3 \\
\hline & & & $71.5^{* *}$ & $63.3 * *$ & $61.7 * *$ & $37.7 * *$ & $35.7 * *$ & $30.0 * *$ & $53.2 * *$ & $50.9 * *$ & $48.2 * *$ \\
\hline & \multirow{4}{*}{$\begin{array}{c}\text { Lowest } \\
\text { combination }\end{array}$} & \multirow{2}{*}{ K78s } & $\mathrm{R} 7$ & R19 & $\mathrm{R} 27$ & $\mathrm{R} 27$ & R45 & R44 & R19 & $\mathrm{R} 27$ & R57 \\
\hline & & & -4.4 & -2.6 & 0.8 & $-21.5^{* *}$ & $-21.4 * *$ & $-20.4 * *$ & -5.0 & -1.6 & 2.3 \\
\hline & & \multirow{2}{*}{ K456s } & R37 & R6 & $\mathrm{R} 35$ & $\mathrm{R} 37$ & R59 & R46 & R37 & R6 & $\mathrm{R} 35$ \\
\hline & & & $-23.9 *$ & -9.5 & -3.6 & $-48.5 * *$ & $-35.4 * *$ & $-25.9 * *$ & $-35.2 * *$ & $-22.9 *$ & -17.9 \\
\hline \multirow{8}{*}{ 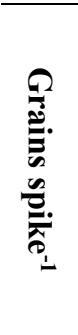 } & \multirow{4}{*}{$\begin{array}{c}\text { Highest } \\
\text { combination }\end{array}$} & & R29 & R31 & R45 & R37 & R6 & R29 & R29 & R31 & $\mathrm{R} 45$ \\
\hline & & & 12.7 & 6.6 & 6.3 & $40.0 * *$ & $19.0 *$ & 7.0 & $152.8 * *$ & $139.0 * *$ & $138.4^{* *}$ \\
\hline & & & $\mathrm{R} 20$ & R37 & $\mathrm{R} 21$ & $\mathrm{R} 20$ & R34 & $\mathrm{R} 21$ & $\mathrm{R} 22$ & $\mathrm{R} 34$ & $\mathrm{R} 20$ \\
\hline & & 0 & $25.7 * *$ & $21.7 * *$ & 13.0 & $88.8 * *$ & $64.8 * *$ & $63.1 * *$ & $127.6 * *$ & $104.7 * *$ & $90.7 * *$ \\
\hline & & & $\mathrm{R} 16$ & $\mathrm{R} 28$ & R60 & $\mathrm{R} 16$ & R56 & $\mathrm{R} 1$ & $\mathrm{R} 16$ & $\mathrm{R} 28$ & R60 \\
\hline & Lowest & & $-53.9 * *$ & $-46.4 * *$ & $-39.7 * *$ & $-53.4 * *$ & $-44.6 * *$ & $-44.5^{* *}$ & 3.4 & 20.2 & 35.2 \\
\hline & combination & & R60 & R31 & $\mathrm{R} 57$ & R60 & R59 & R55 & R60 & $\mathrm{R} 31$ & R57 \\
\hline & & $+50 \mathrm{~s}$ & $-38.6 * *$ & $-30.0 * *$ & $-26.8 * *$ & $-32.2 * *$ & $-21.4 * *$ & $-16.9 *$ & -7.8 & 5.2 & 10.0 \\
\hline
\end{tabular}

Note: " * " and " ** "represent significant differences at $\mathrm{P}<5 \%$ and $\mathrm{P}<1 \%$ levels, respectively.

Table 6. Contribution of lines, testers and their interactions to total variances for 9 agronomic traits.

\begin{tabular}{lccccccccc}
\hline Contribution & Y/P & S/P & TGW & G/S & SL & SN & FSN & SSN & PH \\
\hline Lines & 2.35 & 3.19 & 0.28 & 17.05 & 1.33 & 30.15 & 32.73 & 2.89 & 0.1 \\
Testers & 62.76 & 62.68 & 69.35 & 57.68 & 74.13 & 48.89 & 46.8 & 67.84 & 83.8 \\
Line X tester & 34.88 & 34.13 & 30.37 & 25.26 & 24.54 & 20.97 & 20.47 & 29.27 & 16.1 \\
\hline
\end{tabular}

Note: For characters Y/P represents Yield Plant ${ }^{-1}$, S/P Spikes Plant ${ }^{-1}$, TGW Thousand Grain Weight, G/S Grains Spike ${ }^{-1}$, SL Spike Length, SN Spikelets Number, FSN Fertile Spikelets Number, SSN Sterile Spikelets Number, PHt Plant Height.

\section{CONCLUSION}

The non-additive gene action was predominantly governing all characters studied. The TPSGMS line K456s recorded highest GCA in Y/P, TGW, G/S, SL, SN and FSN. The restorer $2016 Y_{2}-2776$ (R4) and 2016Y2-4117 (R37) showed the highest GCA in five characters. The cross $\mathrm{K} 78 \mathrm{~s} / \mathrm{R} 31$ possessed highest positive SCA values in five characters, while K456s/R2 exhibited highest positive values in six characters. The crosses K78s/R43 and K456s/R21 showed the highest significant positive heterosis estimates over standard check (34.41 and 32.91, respectively), and MP (238.77 and 165.57, respectively) estimates with respect to $\mathrm{Y} / \mathrm{P}$. Also, the crosses $\mathrm{K} 78 \mathrm{~s} / \mathrm{R} 4$ had significant positive heterosis estimates over BP (82.19), MP (211.96) and standard check (23.77) estimates with respect to Y/P. These promising materials could be utilized for parental lines traits improvement and heterosis level enhancement for hybrid wheat breeding program.

\section{ACKNOWLEDGEMENTS}

The financial support by The National Key Research and Development Program of China (2016YFD0101603) is gratefully acknowledged. 


\section{LITERATURE CITED}

Anonymous. 1999. Agrobase 99. Agronomix Software, Inc.171 Waterloo St.Winnipeg, Manitoba, CANADA R3N 0S4 www.agronomix.mb.ca

Anonymous. 2015. High yield of Yunnan hybrid wheat planted in Vietnam, China. Yunnan Daily. Accessed on January 28, 2019 from http://www.chinagrain.cn/xiamai/2015/6/2/20156215405832 549.shtml

Allard, A.M. 1960. Principles of Plant Breeding. Jhon Willy and Sons. Inc. New York, U.S.A., p: 92.

Fasahat, P., A. Rajabi, J.M. Rad and J. Derera. 2016. Principles and utilization of combining ability in plant breeding. Biom. Biostat. Int J. 4(1): 00085.

Fisher, R.A. and F. Yates. 1967. Statistical Tables for Biological, Agricultural and Medical Research, London Group Limited. London.

Fonseca, S. and F.L. Patterson. 1968. Hybrid vigor in a sevenparent diallel crosses in common winter wheat (Triticum aestivum L.). Crop Sci. 8: 85-88.

Gomez, K.A. and A.A. Gomez. 1984. Statistical Procedures for Agriculture Research. John Wiley \& Sons, New York: WileyInterscience.

Hochholdinger, F. and J.A. Baldauf. 2018. Heterosis in plants. Current Biology. 28: 1089-1092.

Istipliler, D., E. Ilker, F. A. Tonk, G. Civi and M. Tosun. 2015. Line $\times$ tester analysis and estimating combining abilities for yield and some yield components in bread wheat. Turk. J. Field Crops. 20: 72-77.

Jain, S.K. and E.V.D. Sastry. 2012. Heterosis and combining ability for grain yield and its contributing traits in bread wheat (Triticum aestivum L.). J. of Agric. and Allied Sci. 1:17-22.

Kearsey, M.J. and H.S. Pooni. 1996. The Genetical Analysis of Quantitative Traits. Chapman and Hall, London.

Kose, A. 2017. Gene action and combining abitiy in line $\times$ tester population of safflower (Carthamus tinctorius L.). Turk. J. Field Crops. 22(2): 197-203.

Krystkowiak, K., T. Adamski, M. Surma and Z. Kaczmarek. 2008. Relationship between phenotypic and genetic diversity of parental genotypes and the specific combining ability and heterosis effects in wheat (Triticum aestivum L.). Euphytica. 165: 419-434.

Mather, K. 1949. Biometrical Genetics. 3rd ed. Cambridge Univ. Press, London, N. Y., 158 p.

Mather, K. and J.L. Jinks. 1982. Biometrical Genetics. 3rd ed. Cambridge Univ. Press, London, N. Y.

Melchinger, A.E. 1999. Genetic Diversity and Heterosis. In: The Genetics and Exploitation of Heterosis and Crop Plants, eds. Coors, J.G. and Stuab, J.E., Crop Science Society of America, Madison, 99-118.

Rasheda, A., M. Muneeb and A. Salam. 2014. Genetic architecture of yield components accessed through line $\times$ tester analysis in wheat (Triticum aestivum L.). Universal J. of Plant Sci. 2(5): 93-96.

Rashid, M., A.A. Cheema and M. Ashraf. 2007. Linextester analysis in basmati rice. Pakistan J. of Botany. 39(6): 20352042.

Saeed, A., M.A. Chowdhry, N. Saeed, I. Khalıq and M.Z. Johar. 2001. Line $\times$ tester analysis for some morpho-physiological traits in bread wheat. Int. J. of Agric. \& Biology. 3(4): 444447.

Salgotra, R.K., B.B. Gupta and S. Praveen. 2009. Combining ability studies for yield and yield components in Basmati rice. An Int. J. on Rice. 46(1): 12-16

Singh, R.K. and B.D. Chaudhary. 1985. Biometrical Methods in Quantitative Genetic Analysis Kalyani; Publisher New Delhi Ludhiana India.

Song, X. B. He, L. Ma, Y. Hu and H. Li. 2005. Genetic study of thermo-sensitive male sterility on thermo-sensitive male sterile wheat line A3314. Scientia Agricultura Sinica. 38(6): 1095-1099.

Steel, R.G.D. and J.H. Torrie. 1980. Principles and Procedures of Statistics. 2nd edition. McGraw Hill Book Company Inc., New York.

Tadesse, W.T., S. Inagaki, M.N. Picard, E. Picard and M. Baum. 2013. Methods and Applications of Doubled Haploid Technology in Wheat Breeding. ICARDA, Aleppo, Syria. 36 p.

Wynne, J.C., D.A. Emery and P.W. Rice. 1970. Combining Ability Estimates in Arachis hypogaea L. II. Field performance of $\mathrm{F}_{1}$ hybrids. Crop Sci. 10(6): 713-715.

Zhao, C. 2013. Research and application of hybrid wheat in China. Engineering Sci. 11(5): 19-21. 